\title{
Dynamical effects in the integrated X-ray scattering intensity from single crystals with microdefects in Bragg diffraction geometry
}

\author{
S. V. Dmitriev, V. B. Molodkin, S. I. Olikhovskii, and V. V. Lizunov \\ G.V. Kurdyumov Institute for Metal Physics, N.A.S. of Ukraine, 36 Academician Vernadsky Blvd., Kyiv, UA-03142, Ukraine
}

dsv2003@ukr.net

The modern microelectronic industry demands the creation of single-crystalline materials having predefined physical properties. It depends on both the perfect crystalline structure of the material and structural defects distributed in subsurface layers or crystal bulk. The technologies of 'defect engineering' are being developed, where the creation of defects or mesoscopic structures is used for targeted change of physical properties of materials. Characterization of such structures by using X-ray diffraction techniques has the important advantages over other methods due to the non-destructive nature of diagnostics and high sensitivity.

The characterization of microdefects in highly perfect crystalline objects requires understanding the influence of dynamical effects of $\mathrm{X}$-ray scattering on the measured diffraction intensities. In particular, of great interest is the occurrence of dynamical effects in the distributions of diffuse scattering intensity that carry the basic information about the imperfections and distortions of a crystal structure.

The aim of the presented work is the theoretical investigation of the dynamical effects in the integrated coherent and diffuse X-ray scattering intensity from imperfect crystals in Bragg diffraction geometry. We considered the sensitivity of the crystal reflectivity integrated with various ways to the characteristics of Coulomb-type defects (Fig. 1).
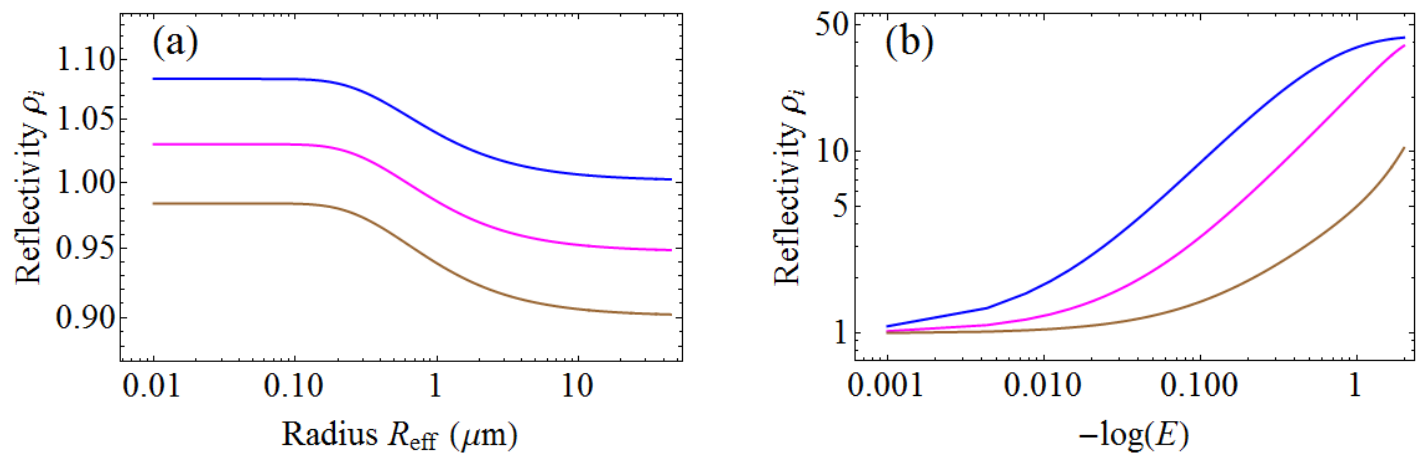

Figure 1. (a) Dependence of the normalized integrated reflection coefficient of imperfect copper crystal, $\rho_{\mathrm{i}}=R_{\mathrm{i}} / R_{\mathrm{i}, \mathrm{dyn}}^{\text {perf }}$, on the effective radius of defects at the static Krivoglaz-Debye-Waller factor equal to $E=0.9,0.95$, and 0.999 (brown, magenta, and blue line, respectively) calculated for the symmetric Bragg diffraction geometry $(\mathrm{Cu}(002), \mathrm{CuK \alpha})$. (b) The same dependence, but on the exponent of the static Krivoglaz-Debye-Waller factor at the effective radius of defects equal to $R_{\mathrm{eff}} / \Lambda_{\mathrm{r}}=0.01,1$, and 10 (blue, magenta, and brown line, respectively, $\Lambda_{\mathrm{r}} \approx 2 \mu \mathrm{m}$ ).

The obtained results showed that in the case of Bragg diffraction geometry the effect of primary extinction is more expressed in both coherent and diffuse scattering. The effect of anomalously weak and strong absorption leads generally to the appearance of asymmetry in differential distributions of the diffraction intensity integrated on one or two dimensions in the reciprocal lattice space. In the diffraction intensity integrated on all the three dimensions, this effect is almost leveled due to mutual compensation of gain and loss in both the coherent diffraction and diffuse scattering intensities due to anomalously weak and strong absorption, respectively. It is established that the extinction effect in diffuse scattering is very sensitive to the ratio between the effective radius of defects and extinction length. The latter depends on the choice of wavelength, order of the reflection, and asymmetry factor. This peculiarity can be used to optimize the choice of the diffraction conditions in the X-ray investigations of different defect types in imperfect crystals. Thus, we can state that accounting for the dynamical effects in the diffuse scattering intensity provides the possibility to significantly enhance the reliability and accuracy of the X-ray diffraction characterization of defects in imperfect crystal structures.

Keywords: dynamical theory of X-ray diffraction; diffuse scattering; microdefects; primary extinction; Bragg diffraction geometry. 